\title{
POTENCIAL DE ATRAÇÃO DE PESSOAS DOS MUNICÍPIOS NA REGIÃO DO SEGUNDO PLANALTO PARANAENSE
}

\author{
Alessandro Vinicios Schneider ${ }^{1 *}$, Roberto Tuyoshi Hosokawa ${ }^{2}$, Romano Timofeiczyk Junior ${ }^{2}$, Ricardo Berger $^{2}$ \\ ${ }^{1 *}$ Universidade Estadual do Paraná, Paranaguá, Paraná, Brasil - schneideravs@ig.com.br \\ ${ }^{2}$ Universidade Federal do Paraná, Curitiba, Paraná, Brasil - roberto.hosokawa@pq.cnpq.br; romano.timo@gmail.com; \\ berger.ufpr@gmail.com \\ Recebido para publicação: 18/11/2014 - Aceito para publicação: 20/06/2016
}

\begin{abstract}
Resumo
Os deslocamentos de passageiros no transporte coletivo intermunicipal de uma determinada região representam um padrão de fluxo ou de interação entre os municípios, sendo que essas relações proporcionam uma dinâmica regional. No estudo observaram-se especificamente os municípios de Telêmaco Borba, Ortigueira, Sapopema, Curiúva, Figueira, São Jerônimo da Serra, Imbaú, Reserva, Tibagi e Ventania. Para a aplicação da modelagem proposta por Isard (1975), se utilizou das distâncias entre os municípios em estudo, suas respectivas populações e os fluxos de passageiros observados, que ocorreram no ano de 2010, no transporte coletivo intermunicipal. Os Fluxos Observados (Iij) no transporte coletivo intermunicipal são diretamente proporcionais ao tamanho das massas (Pi) e inversamente proporcionais a distância que as separa, e pode ser expressa por uma força central, atrativa, hierarquicamente organizada pelo tamanho das populações e as distâncias que as separa. O município que apresentou maior Potencial de Atração $\left(V_{i}\right)$ de pessoas foi Telêmaco Borba, seguido de Reserva, Ortigueira, Tibagi, Curiúva.

Palavras-chave: Potencial de Atração; modelo gravitacional; economia regional.
\end{abstract}

\begin{abstract}
Potential of attraction of people of municipalities in the region of the Parana Second Plateau. Displacements of passengers intercity bus transportation in a region represents a flow pattern or interaction between municipalities, and these relationships provide a regional dynamic. In the study specifically observed the municipalities of Telemaco Borba, Ortigueira, Sapopema, Curiuva, Figueira, São Jeronimo da Serra, Imbau, Reserva, Tibagi and Ventania. For the application of modeling proposed by Isard (1975), we used the distances between the cities under study, their respective populations and passenger flows observed, which occurred in 2010, the intercity public transportation. The Observed Flows $\left(\mathrm{I}_{\mathrm{ij}}\right)$ in intercity public transportation are directly proportional to the size of the masses $\left(\mathrm{P}_{\mathrm{i}}\right)$ and inversely proportional to the distance that separates them, and can be expressed by an attractive central force, hierarchically organized by the size of the populations and distances separates them. The municipality that presented the greatest potential attraction $\left(V_{i}\right)$ was people Telemaco Borba, followed by Reserva, Ortigueira, Tibagi, Curiuva.

Keywords: Potential Attraction; gravitational model; regional economy.
\end{abstract}

\section{INTRODUÇÃO}

O estabelecimento de relações econômicas significativas entre diversas unidades econômicas (setores ou regiões) pressupõem a criação de ligações técnicas, geográficas, humanas ou decisórias entre as unidades, de sorte a proporcionar sua unificação. $\mathrm{O}$ aumento do grau de integração entre tais unidades é efetuado mediante a criação de novas ligações (unidades produtoras, estradas e outros meios de transporte e de comunicação), bem como pelo desenvolvimento da infraestrutura existente.

Estas interações se concretizam por meio dos mais variados fluxos gerados entre elas, como viagens de passageiros, transporte de mercadorias, circulação de jornais e revistas, ligações telefônicas e transmissão de informações e/ou realização de negócios via rede mundial. Isso vem proporcionar um potencial de atração regional ou setorial.

Entre os fatores que estimulam as pessoas a realizar viagens pode-se citar, por exemplo, os deslocamentos para o trabalho, para a escola, para o lazer e para compras. Como fatores que dificultam a realização dos deslocamentos, surgem, por exemplo, o custo das viagens, a distância percorrida e o tempo necessário para realizá-las.

Essas relações proporcionam uma dinâmica regional que, na economia, pode compreender uma área de abrangência, onde compradores e vendedores comercializam seus produtos e serviços a um determinado nível de preço. Dessa maneira, quanto maior a população, maior será a demanda, assim, maiores serão os investimentos em 
produção de bens e serviços, desenvolvimento de produtos, geração de empregos, entre outros fatores, gerando um maior deslocamento de pessoas e, consequentemente, ocasionando um determinado Potencial de Atração $\left(V_{i}\right)$.

Podem-se dar duas interpretações ao conceito de potencial. Por um lado, é uma medida da influência ou do impacto que exercem sobre o ponto " $i$ ", ou seja, o conjunto de massas distribuídas no espaço (incluindo a própria massa de " $i$ "). Por outro lado, é uma medida de acessibilidade do ponto "i" ao conjunto de massas distribuídas no espaço em estudo. Dessa maneira quanto maior for à intensidade ao ponto "i", quando comparado com os outros pontos, maior será seu Potencial de Atração $\left(V_{i}\right)$.

Desta maneira, os deslocamentos de passageiros no transporte coletivo intermunicipal de uma determinada região representam um padrão de fluxo ou de interação entre os municípios. Como consequência, tal fluxo gera um determinado nível de Potencial de Atração $\left(V_{i}\right)$, não levando em conta os fatores que estimulam a realização de viagens, bem como os que impedem ou dificultam a realização das mesmas.

Dentro deste contexto, o Potencial de Atração $\left(V_{i}\right)$ de pessoas decorrente dos fluxos no transporte coletivo intermunicipal pode ser representado por modelos, de modo a ser uma representação simplificada da realidade, sendo que o propósito básico da investigação seria identificar as anormalidades de ordem econômica, social, ambiental, de planejamento urbano e regional de uma determinada região.

Alguns modelos matemáticos, ao longo dos anos, têm sido desenvolvidos e aperfeiçoados, com o intuito de uma melhoria no que se refere à precisão destas estimativas. Nas Ciências Sociais, em comparação com as Ciências Exatas, as dificuldades na construção dos modelos são ainda maiores, pois o comportamento dos indivíduos, às vezes, são inexplicáveis.

Ao longo do século XX, o Modelo Gravitacional desenvolvido por Isaac Newton, foi utilizado nas Ciências Sociais, para explicar fenômenos de imigração e emigração (Leis da Migração), fluxos de comércios entre regiões (Leis da gravitação do varejo), bem como aplicações à engenharia de tráfego da Lei da gravitação de varejo. Isard (1975) adaptou o Modelo Gravitacional de Newton, substituindo as "massas" do modelo original, pelo tamanho das populações, e o raio, pela distância que as separa.

Dentre os clássicos da teoria da localização, é necessário ressaltar a contribuição de Isard (1956; 1975). Inconformado com a interpretação dada pelas teorias econômicas neoclássicas à dimensão espacial, o autor criou, o que passou a se chamar Ciência Regional. A crítica de Isard referia-se ao fato de que a dimensão espacial não era considerada pelos clássicos, os quais se sustentavam na suposição de que deveria ocorrer uma equalização dos preços dos fatores de produção, em função do regime de concorrência perfeita, e da perfeita mobilidade dos fatores, com o que os custos de transporte eram desconsiderados.

A principal contribuição teórica de Isard foi introduzir os problemas de espaço na teoria econômica através do conceito de insumos de distância (o movimento de um peso unitário sobre uma unidade de distância). O preço de um insumo de distância é a taxa de transporte e, como no caso de insumos de capital, uma redução no preço causa um efeito de escala e de substituição.

Isard (1975), em seus artigos e textos, sempre apresentou e desenvolveu técnicas e métodos de análise regional a partir de bases de informação já difundidas em países desenvolvidos, marcadamente os USA, tais como Sistemas de Contas Nacionais, Censos Populacionais, Censos Econômicos, Matrizes de Insumo-Produto, entre outros, mas, preocupava-se explicitamente com indicações e instruções para a construção de tais bases.

Isard (1956) sintetizou a produção teórico-metodológica dessa linha ao fazer a integração do modelo de Von Thünen com a microeconomia (maximização de lucro e minimização de custos). Por meio do conceito de insumos de transporte possibilitou equacionar a questão locacional, apontando o efeito da distância sobre as interações espaciais. Sua obra Locationandspace-economy (1956) provocou o surgimento da ciência regional, que tinha como objetivo melhorar a estrutura espacial e regional das diferentes ciências sociais, especialmente a Economia, mediante o estabelecimento de uma teoria geral mais adequada da localização e do espaço econômico.

Posteriormente, inspirado na célebre Lei da Gravitação Universal, elaborada pelo físico e matemático inglês Isaac Newton em 1687, Isard (1975) observa que o mesmo princípio pode ser empregado na demarcação da área de influência de um polo econômico geograficamente localizado.

O modelo gravitacional proposto por Isard (1975) "permite a definição de um esboço da área de interação de um polo, próximo à ideia de área de mercado, levando em conta o poder de atração determinado positivamente pela intensidade das trocas econômicas e negativamente pela distância geográfica, refletida economicamente no custo de transporte por unidade do produto transportado".

Dentro desse contexto foi concebido o presente artigo, que teve como objetivo geral estimar o Potencial de Atração $\left(V_{i}\right)$ de pessoas dos municípios na região do Segundo Planalto Paranaense.

\section{MATERIAL E MÉTODOS}

No estudo foram contemplados os municípios sendo apresentados em letras arábicas e dispostos em Telêmaco Borba (A), Imbaú (B), Reserva (C), Ortigueira (D), Tibagi (E), Curiúva (F), Ventania (G), Figueira (H), Sapopema (I) e São Jerônimo da Serra (J). A principal razão da escolha dessa região foi que sua economia 
se apresenta de forma diversificada, se destacando o setor madeireiro, tendo como pressuposto, que o município de Telêmaco Borba foi inicialmente impulsionado pela atividade de processamento de madeira para papel e celulose, ali localizada, e posteriormente disseminada a atividade florestal para os municípios vizinhos.

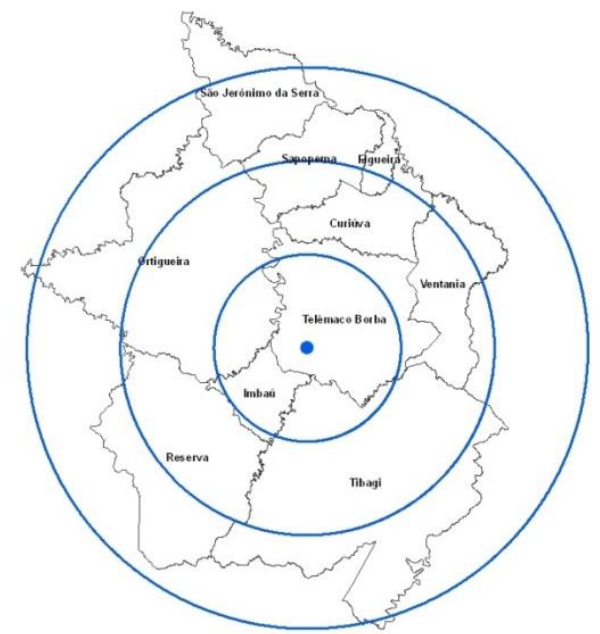

Figura 1. Municípios da região do Segundo Planalto Paranaense.

Figure 1. Municipalities of the Parana Second Plateau.

Essa região foi o caminho das tropas que desbravaram o sul do Brasil, sendo também, destino de garimpeiros, ou seja, a história em comum fez sua parte, mas não foi à única responsável pela construção da identidade dos municípios do Segundo Planalto Paranaense. O fatiamento político teve seu papel importante no desenvolvimento da região, sendo que Castro deu origem a Tibagi, que deu origem a Ventania, a Telêmaco Borba, a Reserva e a Ortigueira. Reserva e Telêmaco Borba deu origem a Imbaú. Jataizinho deu origem a São Jerônimo da Serra que, junto com Congoinhas, deu origem a Curiúva. Este último deu origem a Figueira. São Jerônimo da Serra e Curiúva deram origem a Sapopema.

Segundo Boisier (1980), da mais vasta gama de informações e dados nacionais preparados e publicados regularmente, são selecionados quatro tipos de fontes estatísticas que servem para dar os primeiros passos na matéria de análise regional. Segundo o autor, considera-se, em primeiro lugar, os censos, em segundo lugar as contas nacionais, em terceiro lugar as estatísticas de comércio exterior e, finalmente, as estatísticas de transportes e comunicação.

Para a aplicação da modelagem proposta por Isard (1975), foram utilizadas as distâncias entre os municípios em estudo, suas respectivas populações e os fluxos de passageiros observados, que ocorreram no ano de 2010, no transporte coletivo intermunicipal.

Para compor o modelo gravitacional, os valores das distâncias $\left(\mathrm{d}_{\mathrm{ij}}\right)$ em quilômetros entre os municípios foram fornecidos pelo Departamento de Estradas e Rodagem (DER/PR) e são apresentadas na tabela 1.

Tabela 1. Distâncias $(\mathrm{Km})$ entre os municípios da região Segundo Planalto Paranaense $\left(\mathrm{d}_{\mathrm{ij}}\right)$.

Table 1. Distances (KM) between municipalities of Parana Second Plateau $\left(\mathrm{d}_{\mathrm{ij}}\right)$.

\begin{tabular}{l|rrrrrrrrrr}
\hline Municípios & A & B & C & D & E & F & G & H & I & J \\
\hline A & & 24 & 56 & 54 & 90 & 46 & 85 & 74 & 74 & 104 \\
B & & & 32 & 37 & 27 & 70 & 109 & 98 & 98 & 128 \\
C & & & 79 & 27 & 112 & 151 & 140 & 140 & 170 \\
D & & & & 64 & 107 & 146 & 135 & 135 & 165 \\
E & & & & & & 136 & 46 & 164 & 164 & 194 \\
F & & & & & & & 42 & 27 & 27 & 57 \\
G & & & & & & & & 63 & 69 & 99 \\
H & & & & & & & & & & \\
I & & & & & & & & & & \\
\hline
\end{tabular}

Fonte: Departamento de Estradas e Rodagem (DER/PR), 2010.

No que se refere as "massas" adicionadas ao modelo gravitacional original, foram utilizadas as populações, segundo o censo 2010, fornecidos pelo Instituto Brasileiro de Geografia e Estatística (IBGE), onde Telêmaco Borba possuía 69.872 habitantes, seguido de Reserva com 25.172, Ortigueira (23.380). Os municípios

FLORESTA, Curitiba, PR, v. 46, n. 3, p. 379 - 386, jul. / set. 2016.

Schneider, A. V. et al.

ISSN eletrônico 1982-4688

DOI: $10.5380 /$ rf.v46i3.38601 
menos representativos foram Tibagi (19.344), Curiúva (13.923), São Jerônimo da Serra (11.337), Imbaú (11.274), Ventania (9.957), Figueira (8.293) e Sapopema (6.736) habitantes (IBGE, 2010).

Os dados referentes às viagens realizadas na área de estudo foram obtidos no DER/PR, na forma de relatório contendo dados atualizados, correspondentes ao número de viagens realizadas entre os municípios da região no ano de 2010 .

Tabela 2. Fluxos observados $\left(\mathrm{I}_{\mathrm{ij}}\right)$ de deslocamentos de passageiros no transporte coletivo intermunicipal NA região do Segundo Planalto Paranaense no ano de 2010.

Table 2. Observed flows $\left(\mathrm{I}_{\mathrm{ij}}\right)$ shifts of passenger transport in the region of collective intermunicipal Parana Second Plateau year 2010.

\begin{tabular}{l|rrrrrrrrrr}
\hline Municípios & \multicolumn{1}{|c}{$\mathbf{A}$} & \multicolumn{1}{c}{ B } & \multicolumn{1}{c}{ C } & \multicolumn{1}{c}{ D } & \multicolumn{1}{c}{ E } & \multicolumn{1}{c}{ F } & \multicolumn{1}{c}{ G } & H & \multicolumn{1}{c}{ I } & J \\
\hline A & & 67565 & 7305 & 14328 & 624 & 6880 & 4235 & 878 & 918 & 153 \\
B & 45506 & & 5065 & 6067 & 2118 & 0 & 0 & 0 & 0 & 0 \\
C & 8548 & 5348 & & 0 & 36546 & 0 & 0 & 0 & 0 & 0 \\
D & 12554 & 5731 & 0 & & 0 & 0 & 0 & 0 & 0 & 0 \\
E & 758 & 1557 & 34358 & 0 & & 0 & 201 & 0 & 0 & 0 \\
F & 6364 & 0 & 0 & 0 & 0 & & 810 & 3510 & 7296 & 1029 \\
G & 3776 & 0 & 0 & 0 & 180 & 1353 & & 0 & 0 & 0 \\
H & 1040 & 0 & 0 & 0 & 0 & 3532 & 25 & & 428 & 141 \\
I & 1069 & 0 & 0 & 0 & 0 & 7127 & 0 & 397 & 1198 \\
J & 204 & 0 & 0 & 0 & 0 & 1007 & 0 & 115 & 1235 & \\
\hline
\end{tabular}

Fonte: Departamento de Estradas e Rodagem (DER/PR), 2010.

Observou-se que não ocorreram fluxos em todos os municípios, fato este que pode ser considerado como uma limitação do estudo. A geografia regional proporciona essa descontinuidade, pois o Rio Tibagi corta a região ao meio. Dessa maneira, os municípios a margem direita, não tem ligação direta por meio de transporte coletivo intermunicipal com os municípios da margem esquerdo e vice versa, ou seja, o passageiro precisa fazer uma baldeação, uma troca de ônibus no município de Telêmaco Borba para finalizar o seu deslocamento (Tabela 2).

Entre as 90 possibilidades de fluxos obtiveram-se 43. O restante dos fluxos (47) não ocorre, pois não existem linhas regulares e diretas de transporte coletivo intermunicipal entre esses municípios.

O primeiro passo foi calcular a constante " $k$ ", que representa a média obtida pela relação entre os fluxos observados e a população total da região, conforme expressão abaixo:

$$
\mathrm{k}=\frac{\mathrm{T}}{\mathrm{P}}
$$

em que: $\mathrm{T}=$ Total de fluxos observados; $\mathrm{P}=$ População total; $\mathrm{k}=$ Média de deslocamentos.

Conforme metodologia aplicada por Isard (1975) foi determinado qual o peso da população de cada município nos fluxos totais da região, ou seja, qual a proporção de prováveis deslocamentos conforme a proporção da população de cada município em relação à população total. Com o valor da constante " $k$ " já conhecido, foi calculado o quanto o individuo "i” representa a cidade "i”. Para estimar quais os prováveis deslocamentos do individuo "i”" que terminarão na cidade “j”, utilizou-se a expressão:

$$
T_{i j}=\mathrm{k} *\left(\frac{\mathrm{P}_{\mathrm{i}} * \mathrm{P}_{\mathrm{j}}}{\mathrm{P}}\right)
$$

em que: $\mathrm{T}_{\mathrm{ij}}=$ Deslocamentos prováveis; $\mathrm{k}=$ Média de deslocamentos; $\mathrm{P}=$ População total; $\mathrm{P}_{\mathrm{i}}=$ População de "i”; $P_{j}=$ População de “j”.

Conforme Isard (1975) deve-se, ainda, determinar qual a relação entre os fluxos observados $\left(\mathrm{I}_{\mathrm{ij}}\right)$ e os prováveis fluxos $\left(T_{i j}\right)$. Nesse momento ocorre a primeira relação entre os fluxos observados $\left(\mathrm{I}_{\mathrm{ij}}\right)$ dos passageiros na região e os fluxos prováveis $\left(T_{i j}\right)$.Na expressão anterior não foram considerados todas as variáveis, sendo que o próximo passo foi considerar a distância entre as subáreas, para obter uma melhor estimativa.

Segundo Isard (1975), para observar mais claramente a relevância dessa abordagem, deve-se aplicar os resultados obtidos da relação entre os fluxos observados $\left(\mathrm{I}_{\mathrm{ij}}\right)$ e prováveis $\left(T_{i j}\right)$, em termos de logaritmos, obtendo assim um refinamento da expressão. Em termos matemáticos, utilizou-se a expressão:

$$
\log \frac{\mathrm{I}_{\mathrm{ij}}}{T_{i j}}=\mathrm{a}-\mathrm{b} \log \left(\mathrm{d}_{\mathrm{ij}}\right)
$$

em que: $\mathrm{I}_{\mathrm{ij}}=$ Fluxos observados; $\mathrm{T}_{\mathrm{ij}}=$ Fluxos prováveis; $\mathrm{d}_{\mathrm{ij}}=$ Distância entre os municípios. 
Os coeficientes $b_{0}$ e $b_{1}$ foram obtidos por meio do Método dos Mínimos Quadrados (MMQ). Os parâmetros de ajuste considerados foram o Coeficiente de Determinação $\left(\mathrm{R}^{2}\right)$, o Erro Padrão da Estimativa $\left(\mathrm{S}_{\mathrm{xy}} \%\right)$ e o valor $\mathrm{F}$ ao nível de significância de $5 \%$.

Em seguida foi calculado o valor de "c", o antilog de "a", ou seja, o b obtido por meio da análise de regressão, que, na sua forma natural, assume esta formulação:

$$
\left(\frac{\mathrm{I}_{\mathrm{ij}}}{T_{i j}}\right)=\frac{\mathrm{c}}{\left(\mathrm{d}_{\mathrm{ij}}\right)^{\mathrm{b}}}
$$

em que: $\mathrm{I}_{\mathrm{ij}} / T_{i j}=$ Relação entre os Fluxos Observados $\left(\mathrm{I}_{\mathrm{ij}}\right)$ e os Fluxos Prováveis $\left(T_{i j}\right)$; $\mathrm{c}=\operatorname{antilog}$ de $\mathrm{b}_{0}$; $\mathrm{d}_{\mathrm{ij}}=$ Distância entre os municípios; $b=$ Coeficiente exponencial.

Ao se isolar $\mathrm{I}_{\mathrm{ij}}$, tem-se:

$$
\mathrm{I}_{\mathrm{ij}}=\mathrm{c} \frac{T_{i j}}{\left(\mathrm{~d}_{\mathrm{ij}}\right)^{\mathrm{b}}}
$$

em que: $\mathrm{I}_{\mathrm{ij}}=$ Fluxos Observados; $T_{i j}=$ Fluxos Prováveis; $\mathrm{c}=$ antilog de $\mathrm{b}_{0}$; $\mathrm{d}_{\mathrm{ij}}=$ Distância entre os municípios; $\mathrm{b}$ $=$ Coeficiente exponencial.

E, simplificando ao enunciar $\mathrm{I}_{\mathrm{ij}}$, tem-se:

$$
\mathrm{I}_{\mathrm{ij}}=\frac{C}{\left(\mathrm{~d}_{\mathrm{ij}}\right)^{\mathrm{b}}} * K \frac{\mathrm{P}_{\mathrm{i}} * \mathrm{P}_{\mathrm{j}}}{\mathrm{P}}
$$

em que: $I_{i j}=$ Fluxos Observados; $c=$ antilog de $b_{0}$; $d_{i j}=$ Distância entre os municípios; $b=$ Coeficiente exponencial; $\mathrm{k}=$ Média de deslocamentos; $\mathrm{P}=$ População total; $\mathrm{P}_{\mathrm{i}}=$ População de "i”; $\mathrm{P}_{\mathrm{j}}=$ População de "j".

Como “c", "k" e "P” são constantes, pode-se calcular o valor de "G”, considerado fator de correção, obtido por meio da expressão:

$$
\mathrm{G}=\mathrm{c} * \frac{\mathrm{k}}{\mathrm{P}}
$$

em que: $\mathrm{G}=$ Fator de correção; $\mathrm{c}=$ Antilog de $\mathrm{b}_{0} ; \mathrm{k}=$ Média de deslocamentos; $\mathrm{P}=$ População total.

A constante gravitacional "G" é uma grandeza que não depende de nenhum fator, e não depende do meio onde as massas estão colocadas (ou ausência de meio), ou seja, o seu conteúdo é simples e claro.

No campo da Física, o valor numérico da Constante da Gravitação Universal pode ser determinado por meio experimental, em que, de alguma maneira, a intensidade da força gravitacional que atua sobre cada um dos corpos de massas conhecidas e, que se encontrem a uma distância um do outro, seja conhecido.Dessa maneira, conforme as ideias de Newton, o valor da Força de Atração (F) é diretamente proporcional às massas dos dois corpos e é inversamente proporcional ao quadrado da distância entre os mesmos. As forças aparecem aos pares, ou seja, se um corpo atrai outro, é também atraído pelo primeiro.

No estudo em questão, a constante " $G$ " representa um fator de correção entre às unidades de massas e a distância que a separa. Matematicamente, está vinculada a constante " $k$ ", que representa a média de deslocamento da população, a constante "c", que provem do antilog de $b_{0}$ e a população total (P).

Com todas as variáveis e constantes obtidas por meio desses procedimentos matemáticos, aplicou-se o modelo desenvolvido por Isard (1975), obtendo um novo fluxo estimado pelo modelo gravitacional dos deslocamentos das pessoas por meio do transporte coletivo intermunicipal, tal qual, apresentado a seguir:

$$
I_{i j}=\mathrm{G} \frac{\mathrm{P}_{\mathrm{i} * \mathrm{P}_{\mathrm{j}}}}{\left(\mathrm{d}_{\mathrm{ij}}\right)^{\mathrm{b}}}
$$

em que: $I_{i j}=$ Fluxo estimado; $\mathrm{G}=$ Fator de correção; $\mathrm{P}_{\mathrm{i}}=$ População de "i”; $\mathrm{P}_{\mathrm{j}}=$ População de "j”; $\mathrm{d}_{\mathrm{ij}}=$ distância entre "i" e "j"; $b=b_{1}$ ajustado.

Esta expressão permite estimar o fluxo de pessoas por meio de transporte coletivo intermunicipal na região do Segundo Planalto Paranaense.

Para determinar ou estimar o Potencial de Atração $\left(V_{i}\right)$ dos municípios da região do Segundo Planalto Paranaense, observou-se o Princípio da Superposição, ou seja, quando duas ou mais ondas se propagam,

FLORESTA, Curitiba, PR, v. 46, n. 3, p. 379 - 386, jul. / set. 2016

Schneider, A. V. et al.

ISSN eletrônico 1982-4688

DOI: $10.5380 /$ rf.v46i3.38601 
simultaneamente num mesmo meio e instante e na mesma direção, diz-se que há uma superposição de ondas. A onda resultante é igual à soma algébrica das ondas que cada uma produziria individualmente.

Dessa maneira, esse princípio pode ser aplicado para se obter uma grandeza escalar resultante. Assim, considerando os fluxos de passageiros no transporte coletivo intermunicipal, como ondas, quanto maior for o volume e a intensidade desses fluxos para o mesmo município, maior será o seu Potencial de Atração.

Diante desse contexto, a interação (ou fluxo) entre os pontos " $i$ " e " $j$ " estimada pelo modelo apresentado pode ser calculada de maneira a obter a interação entre " $i$ " com todos os pontos " $j$ ", o que representa o Potencial de Atração do ponto " $i$ ". Cabe dizer, o Potencial de Atração do ponto "i" é igual a sua própria massa $\left(\mathrm{P}_{\mathrm{i}}\right)$, acrescido das massas dos pontos restantes, cada uma corrigida por sua distância a " $i$ ", multiplicada por uma constante (G). Como descrito, a interação entre "i” e " $j$ ” é expressa por:

$$
V_{i}=I_{i 1}+I_{i 2}+I_{i 3}+\cdots+I_{i n}
$$

em que: $V_{i}=$ Potencial de Atração do município " $i$ "; $I_{i l}=$ Fluxo Estimado do município "1" para " $i$ "; $I_{i 2}=$ Fluxo Estimado do município "2" para " $"$ "; $I_{i 3}=$ Fluxo Estimado do município "3" para " $i " ; ~ I i n=$ Fluxo Estimado do município " $n$ " para " $i$ ".

A partir dessa expressão, o Potencial de Atração $\left(V_{i}\right)$ do município pode ser descrito:

$$
V_{i}=\mathrm{G} * \mathrm{P}_{\mathrm{i}}+\mathrm{G} * \frac{\mathrm{P}_{\mathrm{i}} * \mathrm{P}_{1}}{\left(\mathrm{~d}_{\mathrm{i} 1}\right)^{\mathrm{b}}}+\mathrm{G} * \frac{\mathrm{P}_{\mathrm{i}} * \mathrm{P}_{2}}{\left(\mathrm{~d}_{\mathrm{i} 2}\right)^{\mathrm{b}}}+\cdots+\mathrm{G} * \frac{\mathrm{P}_{\mathrm{i}} * \mathrm{P}_{\mathrm{n}}}{\left(\mathrm{d}_{\mathrm{in}}\right)^{\mathrm{b}}}
$$

em que: $V_{i}=$ Potencial de Atração do município “i”; $\mathrm{G}=$ Fator de Correção; $\mathrm{P}_{\mathrm{i}}=$ População de "i”; $\mathrm{P}_{1}=$ População do município 1; $\mathrm{P}_{2}=$ População do município 2; $\mathrm{P}_{\mathrm{n}}=$ População do município $\mathrm{n} ; \mathrm{d}_{\mathrm{i} 1}=$ Distância entre o município " $i$ " e o município " $1 " ; \mathrm{d}_{\mathrm{i} 2}=$ Distância entre o município "i" e o município "2"; $\mathrm{d}_{\mathrm{in}}=$ Distância entre o município " $i$ " e o município " $n$ "; $b=b_{1}$ ajustado.

\section{RESULTADOS E DISCUSSÃO}

Os Fluxos Observados $\left(\mathrm{I}_{\mathrm{ij}}\right)$ de passageiros que se utilizaram do transporte intermunicipal no ano de 2010 totalizou 309.079, dentro de um universo de 199.298 pessoas $\left(\mathrm{P}_{\mathrm{i}}\right)$ que residiam na região. O valor de " $\mathrm{k}$ " calculado foi de 1,55, em média, cada habitante realizou 1,55 viagens dentro da região no ano de 2010. Os Fluxos Prováveis $\left(T_{i j}\right)$ entre as diversas subáreas são apresentados na tabela 3.

Observou-se que, conforme estimativa, os Fluxos Prováveis $\left(T_{i j}\right)$ que ocorreram com maior intensidade foram entre os municípios de Telêmaco Borba e Reserva (13.688), seguido dos Fluxos Prováveis $\left(T_{i j}\right)$ calculados entre os municípios de Telêmaco Borba e Ortigueira (12.713). O que estimou com menor intensidade ocorre entre os municípios de Figueira e Sapopema (435).

Tabela 3. Fluxos prováveis $\left(T_{i j}\right)$ deslocamentos de passageiros no transporte coletivo no Segundo Planalto Paranaense no ano de 2010.

\begin{tabular}{|c|c|c|c|c|c|c|c|c|c|c|}
\hline Municípios & A & B & C & D & $\mathbf{E}$ & $\mathbf{F}$ & $\mathbf{G}$ & H & I & $\mathbf{J}$ \\
\hline $\mathrm{A}$ & & 6130 & 13688 & 12713 & 10519 & 7571 & 5414 & 4509 & 3663 & 6165 \\
\hline B & & & 2209 & 2051 & 1697 & 0 & 0 & 0 & 0 & 0 \\
\hline $\mathrm{C}$ & & & & 0 & 3789 & 0 & 0 & 0 & 0 & 0 \\
\hline D & & & & & 0 & 0 & 0 & 0 & 0 & 0 \\
\hline E & & & & & & 0 & 1499 & 0 & 0 & 0 \\
\hline $\mathrm{F}$ & & & & & & & 1079 & 899 & 730 & 1228 \\
\hline G & & & & & & & & 0 & 0 & 0 \\
\hline $\mathrm{H}$ & & & & & & & & & 435 & 732 \\
\hline I & & & & & & & & & & 594 \\
\hline
\end{tabular}

Table 3. Probable flows $\left(T_{i j}\right)$ shifts in collective passenger transport in Parana Second Plateau year 2010.

Para melhor compreender os resultados, Isard (1975) recomenda realizar procedimento matemático para obter a relação (quociente) entre os Fluxos Observados $\left(\mathrm{I}_{\mathrm{ij}}\right)$ e os Fluxos Prováveis $\left(T_{i j}\right)$, conforme tabela 4.

Com os resultados do logaritmo da relação dos Fluxos Observados $\left(\mathrm{I}_{\mathrm{ij}}\right)$ e os Fluxos Prováveis $\left(T_{i j}\right)$, e os valores da variável distância $\left(\mathrm{d}_{\mathrm{ij}}\right)$, observou-se que os dados permitiram um melhor ajuste. $\mathrm{O}$ coeficiente $\mathrm{b}_{0}$ foi igual a 5,05 e o valor do coeficiente $b_{1}$ foi de $-3,08$. O modelo apresentou o Coeficiente de Determinação $\left(\mathrm{R}^{2}\right)$ igual a 0,72 , assim como um valor elevado para o F de 109,80, e Erro Padrão da Estimativa ( $\left.\mathrm{S}_{\mathrm{xy}} \%\right)$ de $17 \%$. 
Tabela 4. Relação entre os fluxos observados e os fluxos prováveis $\left(\mathrm{I}_{\mathrm{ij}} / T_{i j}\right)$ dos deslocamentos de passageiros no transporte coletivo no ano de 2010.

Table 4. Relationship between observed and probably flows $\left(\mathrm{I}_{\mathrm{ij}} / T_{i j}\right)$ shifts passenger transport collective year 2010.

\begin{tabular}{l|rrrrrrrrrr}
\hline Municípios & $\mathbf{A}$ & $\mathbf{B}$ & $\mathbf{C}$ & $\mathbf{D}$ & $\mathbf{E}$ & $\mathbf{F}$ & $\mathbf{G}$ & $\mathbf{H}$ & $\mathbf{I}$ & $\mathbf{J}$ \\
\hline $\mathrm{A}$ & & 11,02 & 0,53 & 1,13 & 0,06 & 0,91 & 0,78 & 0,19 & 0,25 & 0,02 \\
$\mathrm{~B}$ & 7,42 & & 2,29 & 2,96 & 1,25 & 0 & 0 & 0 & 0 & 0 \\
$\mathrm{C}$ & 0,62 & 2,42 & & 0 & 9,64 & 0 & 0 & 0 & 0 & 0 \\
$\mathrm{D}$ & 0,99 & 2,79 & 0 & & 0 & 0 & 0 & 0 & 0 & 0 \\
$\mathrm{E}$ & 0,07 & 0,92 & 9,07 & 0 & & 0 & 0,13 & 0 & 0 & 0 \\
$\mathrm{~F}$ & 0,84 & 0 & 0 & 0 & 0 & & 0,75 & 3,91 & 10 & 0,84 \\
G & 0,70 & 0 & 0 & 0 & 0,12 & 1,25 & & 0 & 0 & 0 \\
H & 0,23 & 0 & 0 & 0 & 0 & 3,93 & 0,04 & & 0,98 & 0,19 \\
I & 0,29 & 0 & 0 & 0 & 0 & 9,76 & 0 & 0,91 & & 2,02 \\
J & 0,03 & 0 & 0 & 0 & 0 & 0,82 & 0 & 0,16 & 2,08 & \\
\hline
\end{tabular}

Quando realizada a regressão do modelo, a equação obtida foi:

$$
\log \left(\mathrm{I}_{\mathrm{ij}} / T_{i j}\right)=5,0505-3,087 \log \left(\mathrm{d}_{\mathrm{ij}}\right)
$$

A limitação do estudo foi o número de amostras dos fluxos de pessoas no transporte coletivo intermunicipal devido à inexistência de linhas regulares e diretas entre todos os municípios em estudo, diminuindo o "n" amostral do universo. Dessa maneira, proporcionando a diminuição do Coeficiente de Determinação e aumentando o Erro Padrão da Estimativa.

Outro aspecto a ser observado é que a variável (P), isoladamente, não explica totalmente o comportamento das pessoas no que se refere aos deslocamentos no transporte coletivo intermunicipal.

Mesmo diante dessas limitações, pode-se afirmar que o modelo se mostrou consistente, ou seja, representa adequadamente os dados referentes ao comportamento dos fluxos das pessoas no transporte coletivo intermunicipal na região do Segundo Planalto Paranaense, de acordo com o tamanho das populações dos municípios e as distâncias que os separam.

A constante "G", como Fator de Correção, no ano de 2010, foi de 0,87. O modelo gravitacional ajustado pode, então, ser escrito da seguinte forma:

$$
I_{i j}=0,87 \frac{\mathrm{P}_{\mathrm{i}} * \mathrm{P}_{\mathrm{j}}}{\left(\mathrm{d}_{\mathrm{ij}}\right)^{3,08}}
$$

Observou-se que os Fluxos Estimados " $I_{i j}$ " no transporte coletivo intermunicipal deveriam ocorrer em maior quantidade do que foi observado para que ocorresse o equilíbrio entre os dois lados da equação do modelo. Dessa maneira, a constante "G", como Fator de Correção, proporcionou uma diminuição no valor da grandeza do quociente entre o tamanho das massas e a distância que as separa para que ocorresse a igualdade matemática no modelo gravitacional.

No que se refere ao Potencial de Atração $\left(V_{i}\right)$, ou seja, a capacidade de atração de cada município na região, pode ser representado pela equação:

$$
V_{i}=0,87 * \mathrm{P}_{\mathrm{i}}+0,87 * \frac{\mathrm{P}_{\mathrm{i}} * \mathrm{P}_{1}}{\left(\mathrm{~d}_{\mathrm{i} 1}\right)^{3,08}}+0,87 * \frac{\mathrm{P}_{\mathrm{i}} * \mathrm{P}_{2}}{\left(\mathrm{~d}_{\mathrm{i} 2}\right)^{3,08}}+\cdots+0,87 * \frac{\mathrm{P}_{\mathrm{i}} * \mathrm{P}_{\mathrm{n}}}{\left(\mathrm{d}_{\mathrm{in}}\right)^{3,08}}
$$

O município que apresentou o maior Potencial de Atração $\left(V_{i}\right)$ de pessoas foi Telêmaco Borba (61.085; $35,06 \%$ ), seguido do município de Reserva $(22.007 ; 12,63 \%)$. O município de Ortigueira ficou em terceiro lugar com 20.440, representando 11,73\%. Os municípios menos representativos foram Tibagi $(16.913 ; 9,71 \%)$, Curiúva (12.173; 6,99\%), São Jerônimo da Serra $(9.911 ; 5,69 \%)$, Imbaú $(9.861 ; 5,66 \%)$, Ventania (8.705; $5,00 \%)$, Figueira $(7.251 ; 4,16 \%)$ e Sapopema $(5.890 ; 3,38 \%)$.

Observando a configuração da distribuição do Potencial de Atração $\left(V_{i}\right)$ de pessoas dos municípios na região do Segundo Planalto Paranaense, nos remete aos conhecimentos de Chirstaller (1966), onde o autor procurou entender as leis que determinam o número, tamanho e distribuição das cidades, que, segundo ele, são conhecidas como lugares centrais.

Inspirado na célebre Lei da Gravitação Universal, Isard (1975) observou que o mesmo princípio pode ser empregado na demarcação da área de influência de um polo econômico geograficamente localizado. Dessa maneira, permitindo a definição de um esboço da área de interação de um polo, próximo à ideia de área de mercado, levando em conta o poder de atração determinado positivamente pela intensidade das trocas econômicas e negativamente pela distância geográfica.

FLORESTA, Curitiba, PR, v. 46, n. 3, p. 379 - 386, jul. / set. 2016.

Schneider, A. V. et al.

ISSN eletrônico 1982-4688

DOI: $10.5380 /$ rf.v46i3.38601 
Diante dessas inferências e dos resultados apresentados, observou-se que a distribuição espacial dos municípios da região do Segundo Planalto Paranaense, pressupõe um espaço econômico organizado em torno de um núcleo urbano principal. Nesse caso, se caracteriza o município de Telêmaco Borba como núcleo principal e os municípios vizinhos como região complementar, onde se pode observar um comportamento de região nodal ou polarizada.

Na região do Segundo Planalto Paranaense se faz notar a interdependência do crescimento econômico entre os municípios. O município de Telêmaco Borba, como "local central", gerando externalidades positivas nos "municípios entorno". Essa afirmativa se justifica, pois nos municípios de Ventania, Imbaú, Curiúva e Tibagi, "municípios entorno", ocorreu crescimento populacional entre os anos de 1991 e 2010. Fato este, não observado nos "municípios periféricos", São Jerônimo da Serra, Sapopema, Figueira e Ortigueira, onde ocorreu significativa perda de população no mesmo período.

Sob essa ótica, a distribuição espacial da população pode ser considerada como estando num sistema e com as mais variadas ligações funcionais, que proporcionam um sistema hierárquico do Potencial de Atração $\left(V_{i}\right)$ de pessoas, idêntico à distribuição populacional proporcional na região.

Desta maneira, a centralidade proporcional da população de Telêmaco Borba determina a centralidade do Potencial de Atração $\left(V_{i}\right)$ de pessoas, fazendo com que esse potencial seja mais intenso nas regiões mais próximas do centro.

Observou-se que quanto maior a centralidade de um lugar, nesse caso, o município de Telêmaco Borba, maior será o entorno, sendo que os municípios no entorno e menores dependem do município maior, pois quanto mais complexos os serviços oferecidos, maior será a região atendida por esse centro. Assim, quanto maior a população, mais provável que suas taxas de crescimento e seus níveis de desenvolvimento econômico sejam mais elevados, quando comparados aos seus pares.

Dessa maneira, os municípios no entorno, sendo os mais próximos do lugar central, quando comparados com os "municípios periféricos", se beneficiam devido à diminuição do atrito referente à distância entre esses municípios e o lugar central. Já os mais distantes tendem a perdem o Potencial de Atração $\left(V_{i}\right)$ de pessoas decorrente do aumento da distância entre os mesmos.

\section{CONCLUSÕES}

Conclui-se que:

- O modelo gravitacional de Newton, adaptado por Isard, como ferramenta para estimar o Potencial de Atração $\left(V_{i}\right)$ dos municípios do Segundo Planalto Paranaense, por meio dos deslocamentos no transporte coletivo intermunicipal, mesmo tendo princípios mecanicistas das Leis da Física Mecânica, constitui um instrumento operacionalmente útil para avaliar a nodalidade ou causalidade desses eventos. Contudo, não são deterministas, assim o modelo gravitacional não otimizou, mas ao invés, predisse o que é provável que tenha acontecido e, de acordo com a Lei de Gravitação Universal, quanto maior for a população do município maior será seu potencial de atração $\left(V_{i}\right)$ de pessoas;

- Os Fluxos Observados $\left(\mathrm{I}_{\mathrm{ij}}\right)$ no transporte coletivo intermunicipal são diretamente proporcionais ao tamanho das massas $\left(\mathrm{P}_{\mathrm{i}}\right)$ e inversamente proporcionais a distância que as separa, e pode ser expresso por uma força central, atrativa, hierarquicamente organizada pelo tamanho das populações e as distâncias que as separam;

- O município que apresentou maior Potencial de Atração $\left(V_{i}\right)$ de pessoas foi Telêmaco Borba, seguido de Reserva, Ortigueira, Tibagi, Curiúva.

\section{REFERÊNCIAS}

BOISIER, S. Técnicas de análisis regional com información limitada. Cadernos Del ILPES. Instituto Latinoamericano y del Caribe de Planificación Econômica y Social. CEPAL-ILPES/NACIONES UNIDAS. N ${ }^{\circ}$ 27. Santiago de Chile: 1980. ISSN 0020-4080.

CHRISTALLER, W. Central places in southern Germany. New Jersey: Prentice-Hall, 1966.

DEPARTAMENTO DE ESTRADA E RODAGEM (DER/PR). Anuário estatístico do sistema de transporte coletivo regular intermunicipal de passageiros do estado do Paraná. Curitiba, 2010.

INSTITUTO BRASILEIRO DE GEOGRAFIA E ESTATÍSTICA (IBGE). Censo demográfico. Rio de Janeiro, 2010.

ISARD, W. Localizationand Space Economy: A General Theory Relatingto Industrial Location, Market Areas, Land Use, Trade and Urban Structure. Cambridge: MIT Press, 1956.

ISARD, W. Introductionto Regional Science. EnglewoodCliffs, N.J: Prentice-Hall, 1975. 